\title{
Diet and anthropometry at 2 years of age following an oral health promotion programme for Australian Aboriginal children and their carers: a randomised controlled trial
}

\author{
Lisa G. Smithers ${ }^{1 *}$, John Lynch $^{1,2}$, Joanne Hedges ${ }^{3}$ and Lisa M. Jamieson ${ }^{3}$ \\ ${ }^{1}$ School of Public Health, University of Adelaide, Adelaide, SA 5005, Australia \\ ${ }^{2}$ School of Social and Community Medicine, University of Bristol, Bristol BS8 2BM, UK \\ ${ }^{3}$ Indigenous Oral Health Unit, Australian Centre for Population Oral Health, University of Adelaide, Adelaide, SA 5005, Australia \\ (Submitted 19 May 2017 - Final revision received 1 October 2017 - Accepted 18 October 2017 - First published online 4 December 2017)
}

\begin{abstract}
There are marked disparities between indigenous and non-indigenous children's diets and oral health. Both diet and oral health are linked to longer-term health problems. We aimed to investigate whether a culturally appropriate multi-faceted oral health promotion intervention reduced Aboriginal children's intake of sugars from discretionary foods at 2 years of age. We conducted a single-blind, parallel-arm randomised controlled trial involving women who were pregnant or had given birth to an Aboriginal child in the previous 6 weeks. The treatment group received anticipatory guidance, Motivational Interviewing, health and dental care for mothers during pregnancy and children at 6,12 and 18 months. The control group received usual care. The key dietary outcome was the percent energy intake from sugars in discretionary foods (\%EI), collected from up to three 24-h dietary recalls by trained research officers who were blind to intervention group. Secondary outcomes included intake of macronutrients, food groups, anthropometric $z$ scores (weight, height, BMI and mid-upper arm circumference) and blood pressure. We enrolled 224 children to the treatment group and 230 to the control group. Intention-to-treat analyses showed that the \%EI of sugars in discretionary foods was $1.6 \%$ lower in the treatment group compared with control (95\% CI $-3 \cdot 4,0 \cdot 2)$. This culturally appropriate intervention at four time-points from pregnancy to 18 months resulted in small changes to 2-year-old Aboriginal children's diets, which was insufficient to warrant broader implementation of the intervention. Further consultation with Aboriginal communities is necessary for understanding how to improve the diet and diet-related health outcomes of young Aboriginal children.
\end{abstract}

Key words: Indigenous health: Children: Energy intakes: Anticipatory guidance: Motivational Interviewing: Anthropometry

Across the world Indigenous populations suffer health inequities from young ages, compared with non-indigenous populations. In Australia, national surveys indicate that 2-4 year-old Aboriginal and Torres Strait Islander children (respectfully, hereafter referred to as 'Aboriginal') have higher proportions of children with overweight and obesity, and with dental caries than non-Aboriginal children ${ }^{(1-3)}$. These health problems are preventable and share common elements, such as links between sugary foods with adiposity and caries ${ }^{(4,5)}$. Interventions for improving young children's diets are generally designed for the cultural (non-Aboriginal) majority and made available to Aboriginal families but they may not be culturally acceptable ${ }^{(5,6)}$. Often interventions tackle only one health issue (e.g. oral health programmes are implemented independently of other programmes) and are not integrated with health services. This piecemeal approach of the Western medical model is inconsistent with Australian Aboriginal philosophies that incorporate social and spiritual wellbeing, connections with community and country ${ }^{(7)}$.
To our knowledge, there are no culturally appropriate holistic health interventions for families with young Aboriginal children that have dietary outcomes and have been tested via a randomised controlled trial (RCT). Therefore, we developed an oral health promotion intervention for Australian Aboriginal families, from pregnancy until age 2 years. The intervention involved consulting with communities and Aboriginal CommunityControlled Health Organisations to understand the needs and ideas of the community. Broad perspectives that incorporate individual-level, family level and culturally relevant factors was a central tenet of the intervention. The primary outcome was early childhood caries, which will be reported elsewhere. The objective of this paper is to examine diet and anthropometric outcomes because Aboriginal families and the practitioners who care for them have identified concerns about high sugar beverages and nutrition ${ }^{(5,8)}$. The intervention was delivered under the direction and leadership of the study's senior Aboriginal Research Officer (J. H.). Thus, this oral health promotion intervention that built upon broad cultural understandings and

Abbreviations: MI, motivational interviewing; MUAC, mid-upper arm circumference; RCT, randomised controlled trial.

* Corresponding author: L. G. Smithers, email lisa.smithers@adelaide.edu.au 
collaboration with health services offered great potential to influence children's diets and anthropometry.

\section{Methods}

We conducted a single-blind, parallel RCT. The intervention was developed in partnership with local Aboriginal communities and endorsed by an Aboriginal Reference Group who named the trial 'Baby Teeth Talk'. The trial was conducted across metropolitan, rural and remote areas of South Australia (area approximately 1 million $\mathrm{km}^{2}$ ). This trial was conducted according to the guidelines laid down in the Declaration of Helsinki and all procedures involving human subjects were approved by the research ethics committees of the Aboriginal Health Council of South Australia (04-09-362) and University of Adelaide (H-057-2010). The trial was registered in December 2010 at the Australian \& New Zealand Clinical Trial Registry (ACTRN12611000111976; https://www. anzctr.org.au). The protocol has been published ${ }^{(9)}$.

\section{Study design and participants}

Inclusion criteria were women who were South Australian residents, able to provide informed consent and were either pregnant with or gave birth to a baby of Aboriginal ethnicity within the previous 6 weeks. Women/mothers were approached rather than fathers because the intervention commenced during pregnancy. Participants were recruited from hospital antenatal clinics, through Aboriginal Community-Controlled Health Organisations, community centres and by word-ofmouth. Participants could respond directly to flyers (e.g. in antenatal clinics) or were informed by non-study clinic staff during routine antenatal care. Clinic staff then notified trial staff of women interested in being involved. During the course of the trial, six research officers (two Aboriginal ethnicity) approached women and explained the trial using a standard script. Participants provided written informed consent.

\section{Randomisation and blinding}

The randomisation schedule was prepared by a statistician who was not involved in the trial, using a computerised random number generator and random block sizes of 4, 6 and 8. The schedule was stratified by recruitment area: (1) Women's \& Children's Hospital, (2) Flinders Medical Centre and southern metropolitan, (3) Lyell McEwin and northern metropolitan, (4) Whyalla and Port Pirie, (5) Port Augusta and (6) all other areas. Participants were randomly assigned to either the intervention or control groups (1:1 ratio). Allocation to group occurred through a central randomisation service via a computer algorithm, which protected the randomisation schedule. Because of the nature of the intervention, neither participants nor research officers who implemented the intervention were blind to the treatment allocation. To achieve single blinding, a different set of research staff collected 2-year outcome data. Data were also analysed blind.

\section{Intervention}

The intervention was delivered by a research officer during pregnancy and at 6,12 and 18 months, with follow-up at 2 years postpartum. The intervention included anticipatory guidance and Motivational Interviewing (MI) about oral health and diet, as well as dental treatments for families and fluoride varnish for children. Anticipatory guidance involved research officers explaining what to expect as children grows and managing health-related behaviours. MI involved engaging with parents to help them identify and adopt healthier oral health behavioural practices in caring for their child's teeth and diets. The goals and duration of each session was as follows. During pregnancy, the approximately 45 -min session was designed to improve understanding of oral health care during pregnancy and links between poor oral health in the mother and the child. Participants were given a pamphlet, adult toothbrush, adult toothpaste, dental floss and refillable water bottle. One to five dental appointments were organised to reduce pregnant women's oral bacterial load, with costs covered for check-ups, $\mathrm{X}$-rays, scaling, cleaning, restorations and extractions but not cosmetic, orthodontics and endodontics. At 6 months, the goal of the 1-h appointment was to increase mothers understanding of the role of sugary foods and beverages in causing tooth decay. Guidance was given on foods and beverages with high sugar content, and included a focus on children not going to bed at night with a bottle containing a sweet beverage, and working through scenarios for providing healthy choices and dealing with family members who provide sweet foods and beverages to children. Families were given a pamphlet, child toothbrush, child toothpaste, an Aboriginal healthy food cookbook and a fluoride varnish was applied to the child's teeth. At 12-months, the focus of the approximately $1 \mathrm{~h}$ appointment was on increasing understanding of oral hygiene, particularly on how to clean children's teeth, working through scenarios of brushing the teeth of a reluctant child, and brushing teeth when other family members are not supportive. Families were given a pamphlet, child toothbrush, child toothpaste and a children's book on teeth cleaning, and fluoride varnish was applied to the child's teeth. At 18-months, the approximately $45 \mathrm{~min}$ appointment included promoting the child's first visit to a dentist, and included a pamphlet, child toothbrush, child toothpaste, contact details of local dental providers, a children's book on visiting a dentist and fluoride varnish was applied to the child's teeth. Although the specific focus of each session differed, messages about reducing children's intake of sugary foods and cariogenic feeding practices were reinforced at all time-points, and families were free to raise questions and discuss food and beverages at all appointments.

Staff underwent formal MI training before trial implementation, which included attending 2-d basic Motivational Interviewing Training course, an intensive 1-d follow-up course, monthly 1-d follow-up training for 6 months and bi-monthly coaching for another year. All interviews were recorded and a randomly selected subset $41 \%$ were scored by a trained member of the Motivational Interviewing Network of Trainers using a standard procedure and a further twenty interviews were assessed by an international expert for external reliability. The fidelity of the MI component has been published $^{(10)}$ and verified by a member of the Motivational Interviewing Network of Trainers. After data collection at 2 years, 
the control group were offered a delayed version of the intervention to address ethical concerns that children would otherwise not receive any benefit from participation.

\section{Outcomes}

The percentage of total daily energy intake (\%EI) from sugars in discretionary foods is the outcome of interest and is aligned with limiting sugar to reduce caries. Dietary information was collected by $24-\mathrm{h}$ multipass recalls, using a validated protocol for a national survey, which has involved Aboriginal people and parents of 2-year-old children ${ }^{(11)}$. Staff were trained and supported in the 24-h recall method by a nutritionist and dietitian, and asked dietary questions in a culturally sensitive and appropriate manner using a standard script. Up to three recalls were collected, 2 on week days and one on a weekend day. The first recall was collected at a face-to-face appointment and subsequent recalls by telephone. For children who spent time with a carer other than the parent on the day of the recall, we contacted the carer for information about the child's food intake. A food model booklet and standard household measuring cups, spoons, commonly used children's bowls and infant/toddler 'sippy' cups were used to help carers estimate quantities of foods. All diet data were checked and entered into Foodworks (version 7 Pro; Xyris Software) software by a nutritionist or dietitian. Recalls were converted into nutrients using the AUSNUT 2007 database of Australian foods. Discretionary foods were identified using food codes from national health surveys ${ }^{(12)}$. For brevity, the term discretionary foods is applied to beverages, solid and semi-solid foods. Discretionary foods are well defined and include sweet biscuits, cakes, pastries, processed meats, ice creams and ice confections, cream, butter, confectionery, chocolate, fried foods such as potato chips, crisps, salty snacks and sugar-sweetened beverages. Under this method of categorisation, fruit drinks are included as discretionary foods but $100 \%$ fruit juice is considered part of fruit intake (and not discretionary foods). The main dietary outcome was calculated by dividing the energy intake from sugars in discretionary foods by total energy intake.

Secondary outcomes included other nutrients (energy, protein, total carbohydrates, sugars, total fat, MUFA, PUFA, dietary fibre and $\mathrm{Na}$ ), and food groups defined in the Australian Guide to Healthy Eating (AGHE; grain (cereal) foods, fruits, vegetables/legumes/beans, dairy products and alternatives, meat and alternatives). The AGHE food groups were included because of their importance as a nutrition education tool. The Australian government produce an Indigenous version of the AGHE to aid food selection for Aboriginal families. Other secondary outcomes included children's anthropometry and blood pressure. Children's weight, height and mid-upper arm circumference (MUAC) were collected using a standard proto$\mathrm{Col}^{(13)}$. Training for the collection of anthropometric data included a demonstration video produced by the WHO, a written protocol and training days run annually, which included how to correctly measure children's anthropometry. In addition, staff had hands-on training from non-trial participants who were attending a local child care centre (with parental permission). Weight was measured in duplicate to the nearest $10 \mathrm{~g}$ (model
803; Seca; checked monthly using standard weights). Height was measured in duplicate using a portable stadiometer (model 213; Seca) with the child's head positioned in the Frankfort plane. MUAC was measured using a calibrated non-stretchable tape. BMI was calculated as weight $(\mathrm{kg})$ divided by height $\left(\mathrm{m}^{2}\right)$. Weight, height, BMI and MUAC were converted to age- and sexappropriate $z$ scores using the zanthro program in Stata and applying the WHO growth standards as the reference ${ }^{(14,15)}$. BMI categories (thin, healthy, overweight and obese) were calculated using the zbmicat program in Stata which applies Cole et al. cut-points for each BMI category ${ }^{(16,17)}$. Due to small numbers, the thin and healthy BMI categories were combined, and the overweight and obese categories were combined. Blood pressure was collected in duplicate from the child's right arm using an appropriately sized cuff while sitting relaxed on the parent's lap (model HEM-7211; Omron).

\section{Statistical analysis}

A sample size of 400 participants was proposed to detect a $25 \%$ reduction in the trial's primary outcome, early childhood caries ( $80 \%$ power, $\alpha 0.05$ ). The present study is secondary to the main trial and limited to the recruited sample size. Nevertheless, we conducted a post hoc power calculation and estimated that a sample size of 400 participants would enable the detection of a minimum difference in percent energy intake from sugars in discretionary foods of $0.2 \%$ (80\% power, $\alpha 0.05$, mean $10 \cdot 0$ (sD $0 \cdot 7) \%{ }^{(18)}$ ).

Data were analysed according to randomisation group (intention-to-treat) using a statistical analysis plan written a priori. No adjustment was made for the number of analyses. All available data were used in analyses, with main findings from analyses accounting for missing data. Dietary data were analysed by generalised estimating equation (GEE) with adjustment for randomisation strata, week day/weekend and staff member who collected the dietary recall. Multiple recalls were used in the GEE to include day-to-day variance in diet. Children who had no recall data were included in the analysis by weighting. This involved calculating the predicted probability of having no dietary data given the mothers' characteristics at baseline. The inverse of the predicted probability was then used to weight the GEE model $^{(19)}$. Weights were calculated separately by treatment group because different processes may have generated missingness. For example, staff developed rapport with intervention families through repeated contact, whereas the control group had no contact from baseline to 2 years. The results of a sensitivity analysis in which extreme weights were trimmed by $5 \%$ made no difference to the findings (data not shown). Weighting for missing data was considered better than multiple imputation because we had little data to inform an imputation model for 2-year-old diets and because of concerns about introducing bias when imputing missing outcome data for randomised trials ${ }^{(20)}$. As dietary intakes can be misreported, sensitivity analyses were conducted to examine the plausibility of reported energy intakes ${ }^{(21)}$. In sensitivity analyses, children's energy intake from dietary recalls was compared against estimates of their BMR, then upper and lower cut-points of the ratio of energy intake:BMR were applied to identify plausible intakes of energy ${ }^{(22)}$. These analyses are included as online Supplementary material. 
Anthropometric $z$ scores and blood pressure outcomes were analysed by generalised linear models with adjustment for randomisation strata. Blood pressure outcomes also included adjustments for children's height and weight.

Data were analysed using Stata 14.0 (StataCorp).

\section{Results}

Fig. 1 shows the flow of participants through the trial. Recruitment occurred from February 2011 to May 2012. Retention was approximately $65 \%$ in both groups, with $>90 \%$ follow-up. Most attrition was due to difficulties contacting participants who moved homes often, had unreliable telephone access and were attending to family/cultural obligations. Baseline characteristics in Table 1 show high levels of disadvantage experienced by the sample including; high unemployment (treatment 185/223 (83\%), control 190/225(84\%)), smoking during pregnancy $(112 / 223(50 \%), 109 / 225(48 \%))$, health support for financially disadvantaged (172/223(77\%), 181/225(80\%)) and household crowding, as indicated by the number of residents in the home. Age of child at follow-up was similar for both groups; intervention: mean 2.3 (sD 0.3 ) years; control: mean 2.2 (sD 0.3 ) years). Post-randomisation variables such as birth weight and having ever breast-fed were similar between the treatment and control groups (birth weight, treatment: mean 3313 (sD 635) g, control: mean 3263 (sD 669) g; ever breast-fed infant, treatment: 104/224 (46\%) v. control: 108/230 (47\%)).

A total of 508 dietary recalls was collected (263 intervention and 245 control group). Of these, one recall was collected from $138(53 \%)$ intervention and $129(53 \%)$ control participants, two recalls from $76(30 \%)$ and $70(29 \%)$ and three recalls from $49(19 \%)$ and 46 (19\%) participants, respectively. Dietary data are displayed in Table 2 . For the main dietary outcome, intervention group children had $1.6 \%$ lower \%EI from sugars in discretionary foods compared with control $(95 \% \mathrm{CI}-3 \cdot 4,0 \cdot 2$; $P=0.079$ ), and was similar to the complete case analyses $(-1.3 \% ; 95 \%$ CI $-3.1,0.4 ; P=0.133)$; online Supplementary Table S1 and Supplementary Material). This effect was reduced after adjusting for plausible energy intakes (adjusted mean difference $-1 \cdot 2 ; 95 \% \mathrm{CI}-3 \cdot 0$, to $0 \cdot 7 ; P=0 \cdot 210$; see online Supplementary Table S2 for sensitivity analyses of plausible reporters). Intakes of total energy, macronutrients and Na were similar in both groups (Table 2). Likewise, there were negligible differences in major foods groups, except for fruit. Fruit intake was $27 \mathrm{~g}$ higher $(95 \% \mathrm{CI}-0.9,55 ; P=0.07)$ among treatment group children compared with control.

Table 2 shows that intervention children had $0 \cdot 2-0 \cdot 3$ higher point estimates on all anthropometric $z$ scores (weight $0 \cdot 3$;

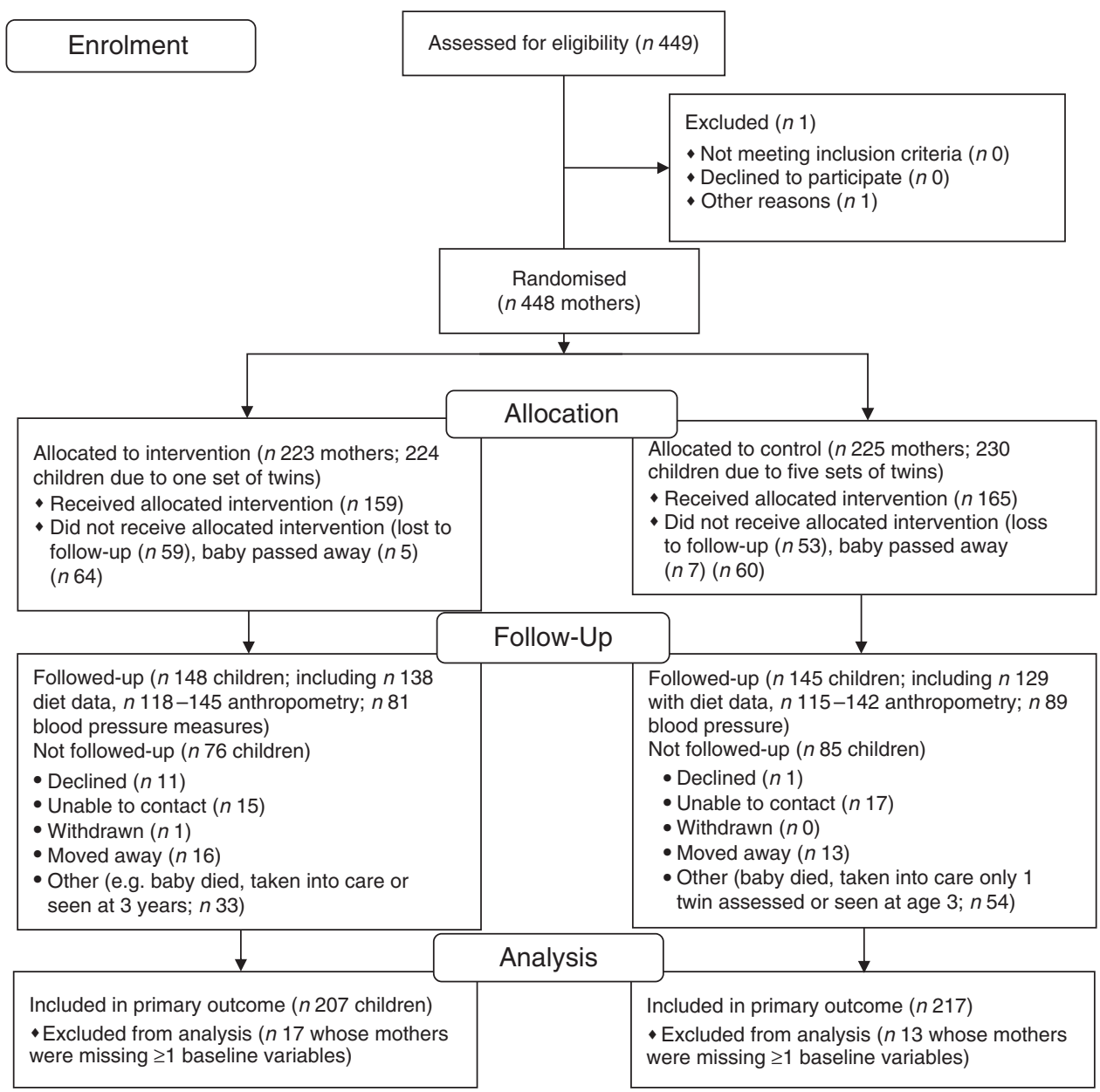

Fig. 1. Flow of participants through the randomised controlled trial. 
Table 1. Characteristics of the study participants (mothers) at baseline (Mean values and standard deviations; numbers and percentages)

\begin{tabular}{|c|c|c|c|c|}
\hline & \multicolumn{2}{|c|}{$\begin{array}{l}\text { Intervention } \\
\quad(n \text { 223) }\end{array}$} & \multicolumn{2}{|c|}{$\begin{array}{l}\text { Control } \\
(n \text { 225) }\end{array}$} \\
\hline & $n$ & $\%$ & $n$ & $\%$ \\
\hline \multicolumn{5}{|l|}{ Maternal age (years) } \\
\hline Mean & \multirow{2}{*}{\multicolumn{2}{|c|}{$\begin{array}{c}24 \cdot 5 \\
5.7\end{array}$}} & \multicolumn{2}{|c|}{$25 \cdot 3$} \\
\hline SD & & & \multicolumn{2}{|c|}{$6 \cdot 1$} \\
\hline Missing & 14 & 6 & 14 & 6 \\
\hline \multicolumn{5}{|l|}{ Maternal ethnicity* } \\
\hline Aboriginal and/or Torres Strait Islander & 187 & 86 & 178 & 79 \\
\hline Other & 29 & 13 & 44 & 20 \\
\hline Missing & 7 & 3 & 3 & 1 \\
\hline \multicolumn{5}{|l|}{ Location } \\
\hline Metropolitan & 106 & 48 & 114 & 51 \\
\hline Regional/remote & 114 & 51 & 109 & 48 \\
\hline Missing & 3 & 1 & 2 & 1 \\
\hline \multicolumn{5}{|l|}{ IRSD quintile* } \\
\hline 1 (most disadvantaged) & 120 & 54 & 126 & 56 \\
\hline 2 & 28 & 13 & 31 & 14 \\
\hline 3 & 30 & 13 & 18 & 8 \\
\hline 4 and 5 (least disadvantaged) & 42 & 19 & 48 & 22 \\
\hline Missing & 3 & 1 & 2 & 1 \\
\hline \multicolumn{5}{|l|}{ Maternal education } \\
\hline High school or less & 159 & 71 & 158 & 70 \\
\hline TAFE or university & 59 & 26 & 64 & 28 \\
\hline Missing & 5 & 2 & 3 & 1 \\
\hline \multicolumn{5}{|l|}{ Employed } \\
\hline Yes & 32 & 14 & 30 & 13 \\
\hline No & 185 & 83 & 190 & 84 \\
\hline Missing & 6 & 3 & 5 & 2 \\
\hline \multicolumn{5}{|l|}{ Car ownership } \\
\hline Yes & 106 & 48 & 118 & 52 \\
\hline No & 111 & 50 & 104 & 46 \\
\hline Missing & 6 & 3 & 3 & 1 \\
\hline \multicolumn{5}{|l|}{ Health Care Card } \\
\hline Yes & 172 & 77 & 181 & 80 \\
\hline No & 39 & 17 & 38 & 17 \\
\hline Missing & 12 & 5 & 6 & 3 \\
\hline \multicolumn{5}{|l|}{ Parity } \\
\hline 0 & 11 & 5 & 15 & 7 \\
\hline 1 & 55 & 25 & 45 & 20 \\
\hline 2 & 41 & 18 & 32 & 14 \\
\hline$\geq 3$ & 38 & 17 & 60 & 27 \\
\hline Missing & 78 & 35 & 72 & 32 \\
\hline \multicolumn{5}{|c|}{ People residing in family home on previous night } \\
\hline $0-1$ & 14 & 6 & 6 & 3 \\
\hline $2-3$ & 83 & 37 & 80 & 36 \\
\hline $4-5$ & 78 & 35 & 84 & 37 \\
\hline $6-7$ & 19 & 9 & 25 & 11 \\
\hline$\geq 8$ & 15 & 7 & 18 & 8 \\
\hline Missing & 14 & 6 & 12 & 5 \\
\hline \multicolumn{5}{|l|}{ Maternal smoking in pregnancy } \\
\hline Never & 47 & 21 & 56 & 25 \\
\hline Used to & 58 & 26 & 57 & 25 \\
\hline Currently & 112 & 50 & 109 & 48 \\
\hline Missing & 6 & 3 & 3 & 1 \\
\hline Alcohol use in pregnancy & & & & \\
\hline Never & 17 & 8 & 18 & 8 \\
\hline Used to & 177 & 79 & 185 & 82 \\
\hline Currently & 22 & 9 & 19 & 8 \\
\hline Missing & 7 & 3 & 3 & 1 \\
\hline
\end{tabular}

IRSD, Index of Relative Socioeconomic Disadvantage; TAFE, Technical and Further Education (trade school/college).

* Categories have been collapsed for confidentiality in accordance with our ethical approval.

95\% CI 0.1, 0.5; $P=0 \cdot 019$; height $0 \cdot 3 ; 95 \%$ CI $0 \cdot 03,0 \cdot 5 ; P=0.028$; MUAC $0 \cdot 2 ; 95 \% \mathrm{CI}-0 \cdot 04,0.5 ; P=0.096$; BMI $0 \cdot 2 ; 95 \% \mathrm{CI}-0.06$, $0 \cdot 4 ; P=0 \cdot 140)$. There were more intervention children in the overweight plus obese BMI category compared with control children (intervention $42 v$. control 34\%; unadjusted risk difference $0.09 ; 95 \% \mathrm{CI}-0.03,0 \cdot 20 ; P=0.137$; adjusted risk difference $0 \cdot 08 ; 95 \% \mathrm{CI}-0 \cdot 04,0 \cdot 19 ; P=0 \cdot 179)$. Equipment malfunction and non-compliance hampered the collection of blood pressure measurements. Nevertheless, the treatment and control groups had similar systolic and diastolic blood pressures (Table 2).

\section{Discussion}

This RCT suggests that a multi-faceted health promotion intervention tailored to Aboriginal families had negligible effects on reducing children's intake of sugars from discretionary foods. The primary findings also reported reductions in caries at 2 years of age, although the effects were small ${ }^{(23)}$. Macronutrient content of treatment and control group diets was similar, and difference according to food groups was small. For example, the increase in fruit intake by the treatment group amounted to approximately 0.2 serves (one serve is about $150 \mathrm{~g}$ ). In the context of the current study, this amounts to increasing fruit intake in the control group children by about one-third ( $27 \mathrm{~g}$ increase $/ 77 \mathrm{~g}$ in control group $=35 \%$ ), although note that we did not separate intake of fruit as a whole food from $100 \%$ juice. The increased intake of fruit and reduction in discretionary foods reflect dietary improvement, and needs to be counterbalanced with the possibility that vegetable and healthy dairy food intakes might be lower. Although no data are available for the \%EI from discretionary foods from other trials, our findings are similar to a 2011-2012 survey of Australian Aboriginal children aged 2-3 years where the proportion of total energy from discretionary foods (which includes sugars in discretionary foods) was $32 \%$ for 2 - to 3-year-old Aboriginal children, and this is approximately $2 \%$ higher than nonAboriginal children ${ }^{(24)}$.

This RCT was intentionally pragmatic in relation to partnerships with Aboriginal Community-Controlled Health Organisations, the frequency of the intervention and the geographic reach across the state. The health-visitor model where staff delivered the intervention at participant's homes (or their nominated location) was appropriate because many participants did not have a car to drive to appointments. Staff built rapport with participants and supported families in a culturally appropriate, non-judgmental manner. A large part of the success of the trial can be attributed to the dedication of the study's Senior Aboriginal Research Officer (J. H.) who became personally known to all study families and was accepted (and by proxy, the rest of the study team) into their lives and wider community. The Aboriginal Research Officer was involved in recruitment, intervention delivery, accompanied other team members when visiting a family for the first time, promoted the study at national celebrations of Indigenous culture, facilitated the follow-up training of the MI component, organised transport to health services and was responsible for dissemination of study results (e.g. presentations to community groups). Having four intervention visits (pregnancy, 6, 12 and 18 months) reflected the balance between health services taking over the intervention (should it be effective), and the timing of important milestones such as the introduction of solid 
Table 2. Comparisons of nutrient, food, anthropometric and blood pressure outcomes of the intervention and control group children at 2 years of age (Mean values and standard deviations; mean differences and $95 \%$ confidence intervals)

\begin{tabular}{|c|c|c|c|c|c|c|c|c|c|c|}
\hline & \multicolumn{2}{|c|}{ Intervention } & \multicolumn{2}{|c|}{ Control } & \multicolumn{3}{|c|}{ Unadjusted $^{*}$} & \multicolumn{3}{|c|}{ Adjusted $†$} \\
\hline & Mean & SD & Mean & SD & Mean difference & $95 \% \mathrm{Cl}$ & $P$ & Mean difference & $95 \% \mathrm{Cl}$ & $P$ \\
\hline $\begin{array}{l}\text { Main dietary outcome: percent energy intake from sugars in discretionary foods } \\
\text { Secondary nutrient-based outcomes (units) }\end{array}$ & $7 \cdot 9$ & 0.6 & $9 \cdot 9$ & 0.7 & -1.9 & $-3.9,-0.04$ & 0.046 & $-1 \cdot 6$ & $-3 \cdot 4,0 \cdot 2$ & 0.079 \\
\hline Total energy $(\mathrm{kJ})$ & 4943 & 146 & 5118 & 183 & -174 & $-634,284$ & 0.457 & -147 & $-575,280$ & 0.500 \\
\hline Protein $(\mathrm{g})$ & $50 \cdot 4$ & $2 \cdot 0$ & 51.4 & $2 \cdot 2$ & $-1 \cdot 0$ & $-6 \cdot 9,4.9$ & 0.732 & $-1 \cdot 8$ & $-7 \cdot 3,3 \cdot 7$ & 0.518 \\
\hline Total carbohydrates (g) & 138.9 & 4.4 & $142 \cdot 8$ & $5 \cdot 7$ & -3.8 & $-17 \cdot 9,10 \cdot 2$ & 0.592 & $-2 \cdot 0$ & $-14 \cdot 7,10 \cdot 6$ & 0.753 \\
\hline Sugar $(g)$ & 68.8 & $2 \cdot 6$ & 70.6 & 3.9 & -1.8 & $-10 \cdot 9,7.4$ & 0.512 & -0.6 & $-9 \cdot 2,8.0$ & 0.888 \\
\hline Starch $(\mathrm{g})$ & 69.5 & $3 \cdot 2$ & 71.6 & $2 \cdot 8$ & $-2 \cdot 1$ & $-10 \cdot 3,6 \cdot 1$ & 0.621 & $-1 \cdot 4$ & $-8 \cdot 9,6 \cdot 1$ & 0.709 \\
\hline Fibre $(\mathrm{g})$ & 11.5 & 0.5 & $11 \cdot 3$ & 0.5 & 0.2 & $-1 \cdot 3,1 \cdot 6$ & 0.834 & 0.002 & $-1 \cdot 3,1 \cdot 3$ & 0.997 \\
\hline Total fat (g) & $45 \cdot 6$ & 1.6 & 48.0 & 1.9 & -2.5 & $-7 \cdot 4,2 \cdot 5$ & 0.328 & $-2 \cdot 2$ & $-7 \cdot 0,2 \cdot 7$ & 0.384 \\
\hline SFA (g) & $21 \cdot 3$ & $0 \cdot 8$ & $22 \cdot 7$ & $1 \cdot 1$ & -1.4 & $-4 \cdot 1,1 \cdot 3$ & 0.310 & $-1 \cdot 3$ & $-3 \cdot 8,1 \cdot 2$ & 0.308 \\
\hline MUFA (g) & $15 \cdot 9$ & 0.6 & $16 \cdot 6$ & 0.7 & -0.7 & $-2 \cdot 6,1 \cdot 2$ & 0.455 & -0.6 & $-2 \cdot 5,1 \cdot 2$ & 0.517 \\
\hline PUFA (g) & $5 \cdot 2$ & 0.3 & 5.5 & 0.2 & -0.4 & $-1 \cdot 1,0.4$ & 0.322 & -0.3 & $-1 \cdot 0,0.4$ & 0.422 \\
\hline $\mathrm{Na}(\mathrm{mg})$ & 1562 & 62 & 1555 & 75 & 6 & $-185,197$ & 0.949 & 8 & $-183,199$ & 0.933 \\
\hline Energy intake from discretionary foods $(\mathrm{kJ})$ & 1667 & 110 & 1922 & 103 & -255 & $-552,41$ & 0.091 & -217 & $-515,79$ & 0.150 \\
\hline Percent El from discretionary foods (\%) & 33.1 & 1.9 & $37 \cdot 0$ & 1.5 & -3.9 & $-8.5,0.7$ & 0.098 & -3.4 & $-8.0,1 \cdot 2$ & 0.152 \\
\hline \multicolumn{11}{|c|}{ Secondary food-based outcomes (food groups are categorised according to the Australian Guide to Healthy Eating) } \\
\hline Grains (g) & 161 & 11 & 158 & 9 & 2 & $-25,30$ & 0.870 & 5 & $-20,30$ & 0.691 \\
\hline Fruits $(\mathrm{g})$ & 104 & 11 & 77 & 10 & 26 & $-2,55$ & 0.069 & 27 & $-0.9,55$ & 0.070 \\
\hline Vegetables $(\mathrm{g})$ & 87 & 9 & 103 & 11 & -17 & $-44,10$ & 0.218 & -15 & $-38,8$ & 0.204 \\
\hline Milk, yogurt, cheese and/or alternatives (g) & 366 & 26 & 392 & 33 & -25 & $-108,57$ & 0.545 & -26 & $-101,49$ & 0.496 \\
\hline Meat and alternatives (g) & 85 & 6 & 86 & 9 & -0.3 & $-21,21$ & 0.974 & -6 & $-28,16$ & 0.603 \\
\hline \multicolumn{11}{|l|}{ Secondary anthropometric and blood pressure outcomesł } \\
\hline Weight $z$ score & 0.9 & $1 \cdot 1$ & 0.6 & 1.0 & 0.3 & $0.1,0.5$ & 0.018 & 0.3 & $0.1,0.5$ & 0.019 \\
\hline Height $z$ score & -0.2 & $1 \cdot 1$ & -0.5 & $1 \cdot 1$ & 0.3 & $0.03,0.5$ & 0.031 & 0.3 & $0.03,0.5$ & 0.028 \\
\hline Mid-upper arm circumference $z$ score & 1.6 & 1.0 & 1.3 & 1.1 & 0.2 & $-0.1,0.5$ & 0.115 & 0.2 & $-0.04,0.5$ & 0.096 \\
\hline BMI $z$ score & 1.4 & $1 \cdot 1$ & 1.2 & 1.0 & 0.2 & $-0.05,0.4$ & 0.123 & 0.2 & $-0.06,0.4$ & 0.140 \\
\hline Systolic blood pressure $(\mathrm{mmHg})$ & 111 & 20 & 114 & 25 & -3 & $-10,3$ & 0.325 & -2 & $-9,5$ & 0.574 \\
\hline Diastolic $(\mathrm{mmHg})$ & 73 & 20 & 77 & 24 & -4 & $-10,3$ & 0.274 & -2 & $-9,4$ & 0.503 \\
\hline
\end{tabular}

* For all dietary analyses, unadjusted mean differences were calculated from a generalised estimating equation that included all available recall data and was weighted by the inverse of the probability of having no $24-\mathrm{h}$ recall data.

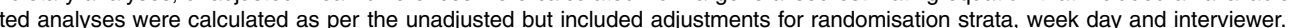

‡ Anthropometry and blood pressure outcomes were analysed by the generalised linear model, with adjustment for randomisation strata (area). Blood pressure outcomes were additionally adjusted for child's height and weight. 
foods at approximately 6 months and teething. All mothers in the intervention group received the same dose of the intervention, although in twelve cases, when a mother was unavailable for the 6-month intervention the components were included in the 12-month session, and in six cases, when a mother was unavailable for the 12-month intervention the components were included in the 18-month session. There was no difference in intervention dose by geographic location. The results indicate that more visits may be necessary to elicit greater dietary and anthropometric benefit, although this would need to be considered against practical and budgetary limitations experienced by health services. The reach across the entire state of South Australia was challenging but similar to government health services. State-wide implementation allows us to judge the overall efficacy across metropolitan, rural and remote regions and across different Aboriginal cultural groups. Together these aspects of the trial represented what we thought would be a feasible intervention to improve general health outcomes of 2-year-old Aboriginal children

We have been unable to identify any comparable health promotion RCT with oral or dietary foci that are implemented from pregnancy and in partnership with indigenous communities. RCT that involve oral health outcomes often do not report dietary data. Examples include an oral health promotional programme among Australian Aboriginal children ${ }^{(25)}$ and a systematic review of community-based interventions for improving 0-18-year-old's oral health ${ }^{(26)}$. Among nonAboriginal parents, a systematic review of MI studies suggest there are benefits to children's dietary (seven studies) or oral health outcomes (four studies), however the evidence base is small for interventions involving children $<2$ years of age (three studies $)^{(27)}$. Reviews of parenting interventions for Aboriginal parents to improve children's health have highlighted a gap in the evidence for rigorously collected nutrition data ${ }^{(28)}$, with only one pilot ( $n$ 43) RCT involving Native American parents. The trial involved sixteen weekly home visits by peer workers and showed children whose parents received nutrition-related parenting support consumed less energy than controls ${ }^{(29)}$. A further three RCT conducted in Australia involve dietary interventions with new parents in the first 2 years of life $\mathrm{f}^{(6,30,31)}$. These trials did not set out to recruit Aboriginal families to an intervention developed by local Aboriginal communities with cultural appropriateness in mind, nor did they report how many Aboriginal participants were enrolled. Therefore, it is not clear how well these interventions might work for Aboriginal families as no one has studied this issue. Nevertheless, all three RCT have results consistent with the current RCT, with all reporting small or null effects. For example, anticipatory guidance with first-time mothers from 6 to 24 months changed the way they fed their infants (less controlling, more responsive), but 24-h dietary recalls showed few differences in the proportion of children consuming discretionary foods ${ }^{(32)}$. As none of these RCT reported the percent energy intake from sugar in discretionary foods, our comparisons are limited.

Of the anthropometric outcomes, intervention children had heavier weight-for-age than controls. As the RCT conducted in other Australian children reported null or small effects on anthropometry ${ }^{(6,30,31)}$ this was unexpected and difficult to explain. The intervention group had higher height-for-age, possibly indicating they are closer to reaching their height potential than controls. However, BMI $z$ scores were also higher suggesting that intervention children were generally heavier than controls. This occurred despite comparable energy intakes in intention-to-treat, complete case and sensitivity analyses adjusted for plausible reporting. As weight-for-age was a secondary outcome in a small sample, follow-up of these children will help determine whether this effect persists over time. Children in both groups had mean $z$ scores indicating heavier weight $(z$ scores $>0$ ) and shorter heights $(z$ scores $<0)$, which may be due to the high proportion of women who smoked during pregnancy (approximately 50\%) and the disadvantaged circumstances in which participants are living ${ }^{(33-35)}$.

Aboriginal community involvement is a strength of the RCT that probably contributed to the excellent follow-up. We invite readers to consider follow-up in context; this RCT was undertaken over vast distances (e.g. travelling $700 \mathrm{~km}$ to the west of South Australia, $400 \mathrm{~km}$ east, $800 \mathrm{~km}$ north), involved highly disadvantaged participants and long 2-year duration. We attribute the follow-up to employment of Aboriginal staff, implementation of an Aboriginal advisory group and proactive engagement with Aboriginal health services. In fact, half of all eligible Aboriginal children born in the state were enrolled (approximately 900 births per annum), demonstrating the widespread community support and generalisability of the results. As it was impossible to conduct the intervention doubleblind, we introduced a second team of researchers to limit bias in the collection of the outcomes. Although all intervention staff were judged as being competent at MI, we have no way of confirming participants receipt and understanding of the trial messages. Participants reported that the dietary recalls were burdensome and this may have increased non-response or socially desirable responses. However, non-response was addressed through analytical modelling. Socially desirable responses would have been attenuated by the 6-month interval between the last anticipatory guidance/MI session and data collection, and different staff collecting outcome data. Furthermore, consistency between the sensitivity analyses (plausible reporters) and main results suggest that socially desirable responses did not unduly influence reporting of energy intake. Other measures of research impact that are valued by Aboriginal communities such as participation empowerment and capacity building were beyond the scope of this paper. For example, the RCT methods applied here do not capture non-academic benefits, perspectives and the perceived value of this programme to Aboriginal people ${ }^{(36)}$.

The small effect of the intervention raises question about how to improve Aboriginal children's health. Community consultation will be undertaken before formal recommendations can be made, which is underway. Options include strategies other than face-to-face sessions, modifying or expanding the content of MI and anticipatory guidance or attempting other promising interventions ${ }^{(37)}$. Although the intervention focused on the mother/main carer, in Aboriginal culture extended family (aunts, uncles, grandparents, cousins) are considered the core family unit, often with many family members caring for the 
child. Furthermore, a strong cultural value is to uphold views of elders, meaning that parents may find it difficult or take more time to change health behaviours. Qualitative analysis of the MI and anticipatory guidance sessions is underway. Preliminary unpublished results suggest parents face day-to-day challenges, conflict and pressures from families and communities regarding what they feed their children. This qualitative research could identify new strategies for improving children's health.

In conclusion, a culturally sensitive health promotion intervention to support parents of Aboriginal children from pregnancy to 18 months did not sufficiently improve children's diets by age 2 years to warrant service-based implementation.

\section{Acknowledgements}

The authors thank the Baby Teeth Talk team including; the Aboriginal Reference Group (Cathy Leane); support of staff from South Australian Aboriginal Community-Controlled Health Organisations; intervention implementation staff (Jessica Merrick); data collection staff (Helen Mills, Jacqueline Aldis, Kostas Kapellas, Reshika Chand and Megan Rebuli); data management support from Kostas Kapellas; and statistical idea for weighting missing data from Murthy Mittinty. The authors acknowledge all of the research officers and support staff who worked on the trial, including those with whom we have been unable to contact and acknowledge by name.

The RCT was funded by an International Collaborative Indigenous Health Research Partnership grant from the National Health and Medical Research Council of Australia (no. 627350). The collection of diet and anthropometric data was partly funded by a Program Grant from the National Health and Medical Research Council of Australia (no. 631947). Colgate Palmolive donated toothpaste, toothbrushes, dental floss and disclosing tablets that was offered as a 'thank you' package to all participants and their families. Neither the grant funding agency nor Colgate Palmolive had any role in the study design, conduct of the study, analysis of data, interpretation of findings or preparation of the manuscript.

L. M. J. designed research (conception); L. M. J., L. G. S. and J. L. developed the research plan, and all authors contributed to study oversight; J. H. conducted research; L. G. S. analysed data and wrote the first draft of the paper and all authors made major contributions to further drafts; L. G. S. and L. M. J. share responsibility for the final content. All authors read and approved the final manuscript.

The authors declare that there are no conflicts of interest.

\section{Supplementary material}

For supplementary material/s referred to in this article, please visit https://doi.org/10.1017/S000711451700318X

\section{References}

1. Jamieson LJ, Parker EJ \& Armfield JM (2007) Indigenous child oral health at a regional and state level. J Pediatr Child Health 43, 117-121.
2. Australian Bureau of Statistics (2013) Australian Aboriginal and Torres Strait Islander Health Survey: First Results, Australia, 2012-2013. Canberra: ABS.

3. Australian Bureau of Statistics (2013) Australian Health Survey: Updated results, 2011-2012. Canberra: ABS.

4. Hooley M, Skouteris H \& Millar L (2012) The relationship between childhood weight, dental caries and eating practices in children aged 4-8 years in Australia, 2004-2008. Pediatr Obes 7, 461-470.

5. Thorpe S, Browne J \& Myers J (2012) Aboriginal Early Years Nutrition \& Physical Activity Needs Assessment Report. Melbourne: Victorian Aboriginal Community Controlled Health Organisation.

6. Campbell KJ, Lioret S, McNaughton SA, et al. (2013) A parentfocused intervention to reduce infant obesity risk behaviors: a randomized trial. Pediatrics 131, 652-660.

7. Australian Government (2013) National Aboriginal and Torres Strait Islander Health Plan 2013-2023, no. 10290]. Canberra: Department of Health and Ageing.

8. Myers J, Thorpe S, Browne J, et al. (2014) Early childhood nutrition concerns, resources and services for Aboriginal families in Victoria. Aust N Z J Public Health 38, 370-376.

9. Merrick J, Chong A, Parker E, et al. (2012) Reducing disease burden and health inequalities arising from chronic disease among Indigenous children: an early childhood caries intervention. BMC Public Health 12, 323.

10. Jamieson L, Bradshaw J, Lawrence H, et al. (2016) Fidelity of motivational interviewing in an early childhood caries intervention involving indigenous Australian mothers. $J$ Health Care Poor Underserved 27, 125-138.

11. Department of Health and Ageing (2007) Australian National Children's Nutrition and Physical Activity Survey. User Guide. Canberra: Australian Government.

12. Australian Bureau of Statistics (2014) Australian Health Survey: Nutrition First Results - Foods and Nutrients, 2011-12. Canberra: ABS.

13. de Onis M, Onyango AW, Van den Broek J, et al. (2004) Measurement and standardization protocols for anthropometry used in the construction of a new international growth reference. Food Nutr Bull 25, S27-S36.

14. World Health Organization (2006) WHO Child Growth Standards: Length/Height-for-Age, Weight-for-Age, Weightfor-Length, Weight-for-Height and Body Mass Index-for-Age: Methods and Development. Geneva: WHO.

15. World Health Organization \& Nutrition for Health (2007) WHO Child Growth Standards: Head Circumference-for-Age, Arm Circumference-for-Age, Triceps Skinfold-for-Age and Subscapular Skinfold-for-Age: Methods and Development. Geneva: WHO.

16. Cole TJ, Bellizzi MC, Flegal KM, et al. (2000) Establishing a standard definition for child overweight and obesity worldwide: international survey. BMJ 320, 1240-1243.

17. Cole TJ, Flegal KM, Nicholls D, et al. (2007) Body mass index cut offs to define thinness in children and adolescents: international survey. BMJ 335, 194.

18. Rangan AM, Randall D, Hector DJ, et al. (2008) Consumption of 'extra' foods by Australian children: types, quantities and contribution to energy and nutrient intakes. Eur J Clin Nutr 62, 356-364.

19. Carpenter JR, Kenward MG \& Vansteelandt S (2006) A comparison of multiple imputation and inverse probability weighting for analyses with missing data. $J R$ Stat Soc Ser $A$ 169, 571-584.

20. Sullivan TR, Salter AB, Ryan P, et al. (2015) Bias and precision of the 'multiple imputation, then deletion' method for dealing with missing outcome data. Am J Epidemiol 182, 528-534.

21. Rangan AM, Flood VM \& Gill TP (2011) Misreporting of energy intake in the 2007 Australian Children's Survey: identification, 
characteristics and impact of misreporters. Nutrients 3 186-199.

22. Black AE (2000) Critical evaluation of energy intake using the Goldberg cut-off for energy intake:basal metabolic rate. A practical guide to its calculation, use and limitation. Int J Obes 24, 1119-1130.

23. Jamieson L, Hedges J, Parker E, et al. (under review) Efficacy of an early childhood caries intervention among Indigenous Australian children. Community Dent Oral Epidemiol.

24. Australian Bureau of Statistics (2015) Australian Aboriginal and Torres Strait Islander Health Survey: Nutrition Results Food and Nutrients, 2012-13. Canberra: ABS.

25. Slade GD, Balilie RS, Roberts-Thomson K, et al. (2011) Effect of health promotion and fluoride varnish on dental caries among Australian Aboriginal children: results from a community-randomized controlled trial. Community Dent Oral Epidemiol 39, 29-43.

26. de Silva AM, Hegde S, Nwagbara BA, et al. (2016) Communitybased population-level interventions for promoting child oral health. Cochrane Database Syst Rev 9, CD009837.

27. Borrelli B, Tooley EM \& Scott-Sheldon LAJ (2015) Motivational interviewing for parent-child health interventions: a systematic review and meta-analysis. Pediatr Dent 37, 254-265.

28. Bowes J \& Grace R (2014) Review of Early Childhood Parenting, Education and Health Intervention Programs for Indigenous Children and Families in Australia. Closing the Gap Clearninghouse. Canberra: Australian Institute of Health and Welfare \& Australian Institute of Family Studies.

29. Harvey-Berino J \& Rourke J (2003) Obesity prevention in preschool native-American children: a pilot study using home visiting. Obes Res 11, 606-611.
30. Wen LM, Baur LA, Simpson JM, et al. (2012) Effectiveness of home based early intervention on children's BMI at age 2: Randomised controlled trial. BMJ 344, 1-11.

31. Daniels LA, Mallan KM, Nicholson JM, et al. (2013) Outcomes of an early feeding practices intervention to prevent childhood obesity. Pediatrics 132, e109-e118.

32. Daniels LA, Mallan KM, Battistutta D, et al. (2014) Child eating behavior outcomes of an early feeding intervention to reduce risk indicators for child obesity: the NOURISH RCT. Obesity (Silver Spring) 22, E104-E111.

33. Muraro AP, Gonçalves-Silva RM, Moreira NF, et al. (2014) Effect of tobacco smoke exposure during pregnancy and preschool age on growth from birth to adolescence: a cohort study. BMC Pediatr 14, 99.

34. Matijasevich A, Brion MJ, Menezes AM, et al. (2011) Maternal smoking during pregnancy and offspring growth in childhood: 1993 and 2004 Pelotas cohort studies. Arch Dis Child 96, 519-525.

35. Grzeskowiak LE, Hodyl NA, Stark MJ, et al. (2015) Association of early and late maternal smoking during pregnancy with offspring body mass index at 4 to 5 years of age. J Dev Orig Health Dis 6, 485-492.

36. Bainbridge R, Tsey K, McCalman J, et al. (2015) No one's discussing the elephant in the room: contemplating questions of research impact and benefit in Aboriginal and Torres Strait Islander Australian health research. BMC Public Health 15, 969.

37. Black AP, Vally H, Morris PS, et al. (2013) Health outcomes of a subsidised fruit and vegetable program for Aboriginal children in northern New South Wales. Med J Aust 199, 46-50. 